\title{
Towards 400GBASE 4-lane Solution Using Direct Detection of MultiCAP Signal in 14 GHz Bandwidth per Lane
}

Iglesias Olmedo, Miguel; Tianjian, Zuo; Jensen, Jesper Bevensee; Qiwen, Zhong; Xu, Xiaogeng; Tafur Monroy, Idelfonso

\section{Published in:}

2013 Optical Fiber Communication Conference and Exposition and the National Fiber Optic Engineers Conference (OFC/NFOEC)

Link to article, DOI:

10.1364/OFC.2013.PDP5C.10

Publication date:

2013

Document Version

Publisher's PDF, also known as Version of record

Link back to DTU Orbit

Citation (APA):

Iglesias Olmedo, M., Tianjian, Z., Jensen, J. B., Qiwen, Z., Xu, X., \& Tafur Monroy, I. (2013). Towards 400GBASE 4-lane Solution Úsing Direct Detection of MultiCAP Signal in 14 GHz Bandwidth per Lane. In 2013 Optical Fiber Communication Conference and Exposition and the National Fiber Optic Engineers Conference (OFC/NFOEC) (pp. PDP5C.10). IEEE. https://doi.org/10.1364/OFC.2013.PDP5C.10

\section{General rights}

Copyright and moral rights for the publications made accessible in the public portal are retained by the authors and/or other copyright owners and it is a condition of accessing publications that users recognise and abide by the legal requirements associated with these rights.

- Users may download and print one copy of any publication from the public portal for the purpose of private study or research.

- You may not further distribute the material or use it for any profit-making activity or commercial gain

- You may freely distribute the URL identifying the publication in the public portal 


\title{
Towards 400GBASE 4-lane Solution Using Direct Detection of MultiCAP Signal in $14 \mathrm{GHz}$ Bandwidth per Lane
}

\author{
Miguel Iglesias Olmedo $^{(1)}$, Zuo Tianjian ${ }^{(2)}$, Jesper Bevensee Jensen ${ }^{(1)}$, Zhong Qiwen ${ }^{(2)}$, Xu Xiaogeng ${ }^{(2)}$, \\ Idelfonso Tafur Monroy ${ }^{(1)}$ \\ ${ }^{(1)}$ DTU Fotonik, Department of Photonics Engineering, Technical University of Denmark, DK2800 Kgs. Lyngby, Denmark \\ ${ }^{(2)}$ Transmission Technology Research Department, Huawei Technologies Co., Ltd., Shenzhen, 518129, China \\ molm@fotonik.dtu.dk
}

\begin{abstract}
We report on an experimental demonstration of $102 \mathrm{Gbit} / \mathrm{s}$ transmission over a 15km single wavelength and polarization fiber link with $14 \mathrm{GHz} 3 \mathrm{~dB}$ bandwidth. Novel multi-band CAP signaling allows for a 4-lane 400GBASE long reach solution. OCIS codes: (060.2330) Fiber optics communications; (060.4080) Modulation
\end{abstract}

\section{Introduction}

Data center links operating at lane rates of $100 \mathrm{Gbit} / \mathrm{s}$ per wavelength are required in order to cope with future demands of bandwidth. In addition, there is an emerging need for increasing the reach up to $10 \mathrm{~km}$, forcing $100 \mathrm{Gbit} / \mathrm{s}$ solutions to be robust to dispersion while keeping complexity low. By retaining a signal bandwidth of less than $28 \mathrm{GHz}$ and using advanced multi-band, multi-level modulation format, we demonstrate experimentally a $15 \mathrm{~km}, 102 \mathrm{Gbit} / \mathrm{s}$ single wavelength and single polarization data link for extended reach client side.

Link capacities as high as $400 \mathrm{Gbit} / \mathrm{s}$ and even $1.6 \mathrm{Tbit} / \mathrm{s}$ are already projected as potential next steps. Current and upcoming standards for $100 \mathrm{Gbit} / \mathrm{s}$, such as 100GBASE-SR10, 100GBASE-SR4, and 100GBASE-LR4 are based on using 10 lanes of $10 \mathrm{Gbit} / \mathrm{s}$ or 4 lanes at $25 \mathrm{Gbit} / \mathrm{s}$ each. Traditionally, the strategy for capacity upgrades has been to exploit the benefits of parallel optics and to rely on higher bandwidth availability for the electronic and optical components. However, this approach would require e.g. 16 lanes at $25 \mathrm{Gbit} / \mathrm{s}$ in order to achieve the $400 \mathrm{Gbit} / \mathrm{s}$ target, thereby making it challenging to meet $400 \mathrm{Gbit} / \mathrm{s}$ form-factor pluggable (e.g. CDFP2 and CDFP4) requirements on power consumption and footprint [1]. Therefore, it is crucial to develop other solutions for beyond $100 \mathrm{Gbit} / \mathrm{s}$ data links satisfying these industry requirements in terms of footprint, power consumption and cost efficiency.

Advanced modulation formats have gained increasing interest from research as well as industry as a method to reduce the number of lanes while increasing the total link capacity. Recent reported experiments include 112 Gbit/s half cycle - 16 level quadrature amplitude modulation (QAM) [2], and 100 Gbit/s, 25 Gbaud 4 level pulse amplitude modulation (PAM) [3]. Discrete multi tone modulation (DMT) has also recently been demonstrated to achieve $100 \mathrm{Gbit} / \mathrm{s}$ [4]. However, all mentioned approaches require either dual polarization or a wavelength division multiplexing (WDM) scheme to achieve the claimed bitrates, and thus doubles the number of lanes and light sourcephoto detector pairs required in the system.

This paper reports on a feasible solution for the possible upcoming $400 \mathrm{Gbit} / \mathrm{s}, 4$ lane standards targeting $2 \mathrm{~km}$ to $10 \mathrm{~km}$ reach applications. The proposed scheme employs four $100 \mathrm{Gbit} / \mathrm{s}$ single wavelength, single polarization lanes. An experimental demonstration of a single lane with optical transmission over $15 \mathrm{~km}$ standard single mode fiber (SSMF) in the O-band has been carried out. A $102 \mathrm{Gbit} / \mathrm{s}$ signal using a novel multi-band carrierless amplitude phase modulation (MultiCAP) signal is successfully generated, transmitted and detected employing a link with an end-to-end $3 \mathrm{~dB}$ bandwidth of only $14 \mathrm{GHz}$. This experimental breakthrough is enabled by the combination of a novel signaling format and the use of digital signal processing techniques.

To the best of the authors' knowledge, this approach achieves the highest experimental reported bit rate using CAP modulation in a single wavelength, single polarization, and direct detection optical link. By the possible extension to 4 lanes, these results demonstrate the prospect for 400GBASE solutions with more than $10 \mathrm{~km}$ reach.

\section{Principle of operation for multi-band CAP}

Carrierless amplitude phase modulation (CAP) is a multidimensional and multi-level modulation scheme proposed in mid 70's by Falconer et. al. at Bell Labs [5]. CAP displays certain similarities to quadrature amplitude modulation (QAM) in its ability to transmit two streams of data in parallel. In contrast to QAM, however, CAP does not rely on a carrier, but uses filters with orthogonal waveforms to separate the different data streams. Although CAP, potentially, can support more than two dimensions, in its most fundamental form (2D-CAP), only two dimensions are used, and the two orthogonal waveforms are constructed by multiplying a square root raised cosine pulse with a sine and a cosine function respectively. 


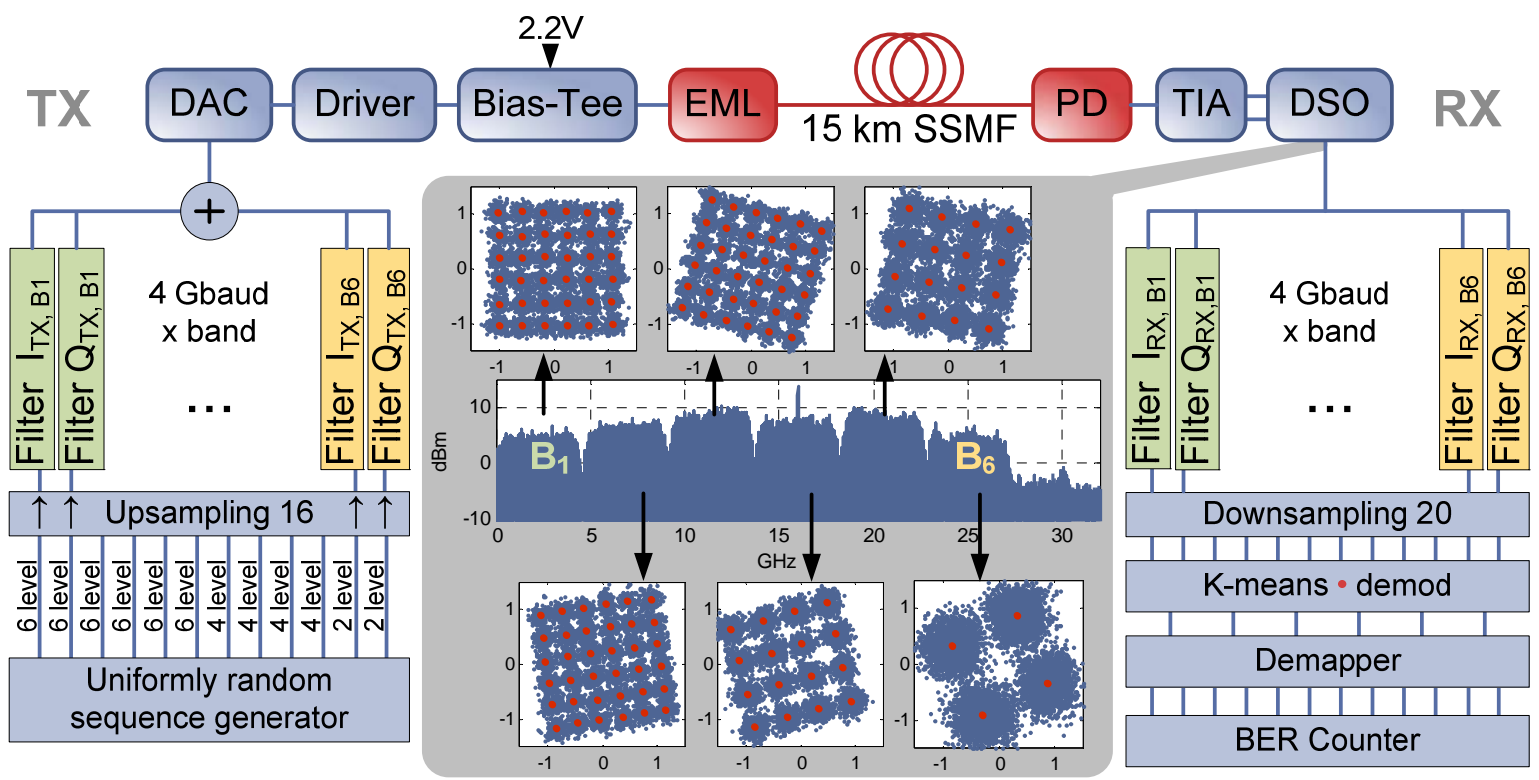

Fig. 1: Setup. Digital to analogue converter (DAC), externally modulated laser (EML), photodiode (PD), digital storage oscilloscope (DSO).

In this paper, we propose a novel, modified 2D-CAP modulation format termed MultiCAP, and employ it to achieve a bit rate of $102 \mathrm{Gbit} / \mathrm{s}$. Instead of using a single 25-Gbaud, 16-level 2D-CAP signal to achieve $100 \mathrm{Gbit} / \mathrm{s}, 6$ sub bands are constructed, each of them modulated at a symbol rate of 4 Gbaud. By breaking the signal into sub bands, the modulation order and signal power can be tailored to the signal to noise ratio (SNR) in each sub band. This effectively overcomes the greatest drawback of conventional CAP, namely the need of a flat frequency response of the channel. An additional advantage of MultiCAP in systems employing DSP for the signal generation is a relaxation of the requirement for the digital to analogue converter (DAC). For a conventional CAP signal, a minimum up sampling factor of three samples per symbol is required for acceptable performance, resulting in a total sampling rate of $75 \mathrm{GSa} / \mathrm{s}$ for a $100 \mathrm{Gbit} / \mathrm{s} 16$ level CAP signal at $25 \mathrm{Gbaud}$. As the symbol rate in each sub band for a MultiCAP signal is much lower, a high up sampling factor can be used while the required sampling rate is kept at a minimum. In comparison to DMT, CAP is shown to offer advantages in SNR requirements and robustness to multipath interferences[6]. Additionally, (de)modulation can be implemented using electrical filters without the need for carrier recovery, frames or adaptive equalization. In our experimental demonstration, these features enable us to generate a 102 Gbit/s 6-band MultiCAP signal using a DAC with a sampling rate of $64 \mathrm{GSa} / \mathrm{s}$, and transmit it over a channel with a $3 \mathrm{~dB}$ bandwidth as low as $14 \mathrm{GHz}$.

\section{Experimental setup}

The MultiCAP operation principle and the setup used in the experimental demonstration is illustrated in Fig. 1. The main building blocks are a transmitter comprising a DAC, a driver amplifier, a bias-tee and an externally modulated laser (EML); a $15 \mathrm{~km}$ SSMF link; and a receiver consisting of a PIN photodetector with a transimpedance amplifier (TIA) and an $80 \mathrm{GSa} / \mathrm{s}$ digital storage oscilloscope. Signal generation and demodulation is performed off-line using Matlab.

For the signal generation, 12 uniformly distributed data sequences with a length of 16384 symbols are generated with modulation orders from 2 to 6 according to the desired modulation orders in the individual MultiCAP sub bands. The 12 symbol sequences are up sampled to $16 \mathrm{Sa}$ /symbol and filtered by the 6 pairs of MultiCAP sub band transmitter filters. The filters are finite impulse response (FIR) filters with a length of 10 symbols each. The combined 102 Gbit/s MultiCAP signal is generated by simply adding the outputs of the 6 filter pairs. By adjusting the weights of each pair of filters, the non-flat frequency response of the channel is pre-compensated. The signal generation is performed in Matlab, and used to drive a $64 \mathrm{GSa} / \mathrm{s}$ DAC with an effective resolution of 5 bits.

The DAC output is amplified to a peak-to-peak voltage of $2 \mathrm{~V}$ and used to drive a 1293.55 nm integrated distributed feedback laser - electro absorption modulator (DFB-EAM) with the 3-dB bandwidth of $20 \mathrm{GHz}$. The signal from the DFB-EAM is propagated through a $15 \mathrm{~km}$ SSMF link with a total link loss of $7 \mathrm{~dB}$. Launch power is $5 \mathrm{dBm}$. The optical spectrum back-to-back (B2B) and after transmission is shown in Fig. 3. The end-to-end channel frequency response is measured by performing a discrete frequency sweep with the DAC and shown in Fig. 4 along 


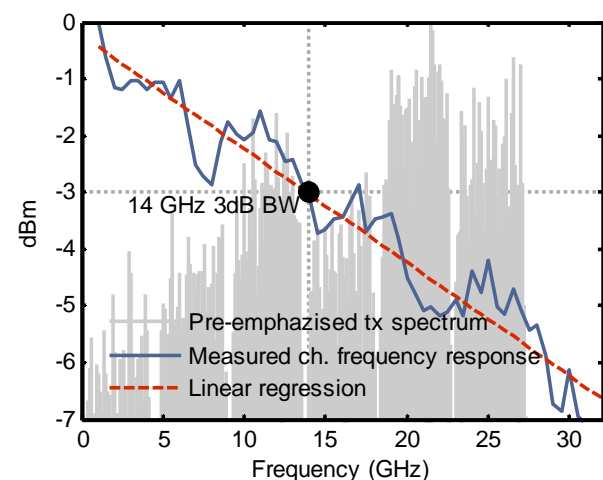

Fig. 2: Frequency response pre-DAC signal spectrum

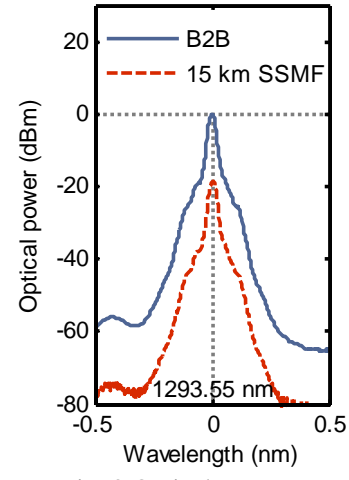

Fig. 3:Optical spectrums

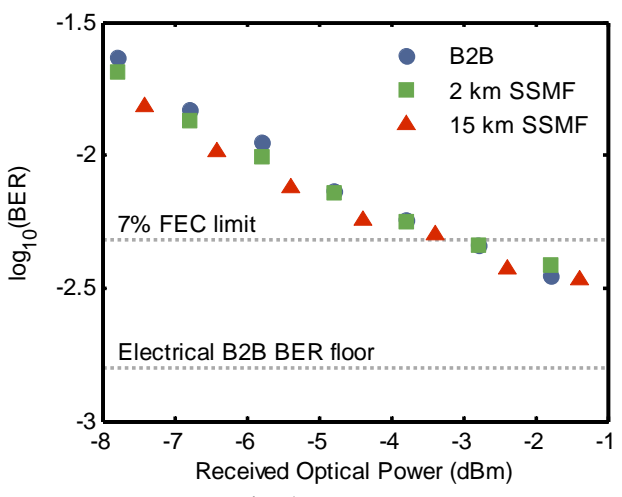

Fig. 4: BER curves

with the spectrum of the pre-compensated 6-band MultiCAP signal. We can observe that the 3-dB bandwidth of the channel is $14 \mathrm{GHz}$, while the signal occupies a total bandwidth of $28 \mathrm{GHz}$.

After photodetection, the signal is sampled and stored by the DSO for off-line processing. The signal is demodulated by filtering with a time inverted version of the transmitter filters. After filtering, the signals are down sampled, and the two orthogonal components of the 6 bands can be obtained to construct the received constellation diagrams shown as inserts in Fig. 1 together with the received spectrum. Demodulation and compensation for constellation rotation and asymmetry caused by local non-flat in-band spectral response is performed employing the K-means algorithm [7].

\section{Results}

Fig. 2 shows the measured bit error ratio (BER) as a function of the received optical power B2B and after $2 \mathrm{~km}$ and $15 \mathrm{~km}$ SSMF transmission. Receiver sensitivity at the 7\%-overhead forward error correction (FEC) limit of $4.810^{-3}$ is $-4.3 \mathrm{dBm}$ in all cases, and no signal degradation or power penalty is observed from the transmission. Due to the limited effective resolution of the DAC [8], a BER floor of the electrical signal driving the EML is measured at $1.5 \cdot 10^{-3}$.

The advantages of the MultiCAP approach include ability for channel response pre-compensation, reduced DAC sampling rate requirements, and tailoring of the modulation order to the SNR of the individual sub bands is clearly observed, as these are exactly the features that enable the generation of the $102 \mathrm{Gbit} / \mathrm{s}$ signal using a $64 \mathrm{GSa} / \mathrm{s} \mathrm{DAC}$ and transmitting it over an channel with an end-to-end $3 \mathrm{~dB}$ bandwidth of $14 \mathrm{GHz}$.

\section{Conclusions}

A novel approach named MultiCAP has been employed to demonstrate a $15 \mathrm{~km}$ optical link with a total bit rate of $102 \mathrm{Gbit} / \mathrm{s}$ using only a single wavelength and direct detection. In this reported experiment, assuming FEC encoding an effective bit rate of $95.36 \mathrm{Gbit} / \mathrm{s}$ is achieved. Despite the use of a high speed (64 GSa/s) DAC, the signal generation relies on the use of transversal filters in order to maintain a level of simplicity in the digital signal processing. By extending these results to four lanes, the prospects of $400 \mathrm{Gbit} / \mathrm{s}$ optical interconnect have been demonstrated for next generation client side data links.

\section{References}

[1] C. Cole, "Beyond 100G client optics," IEEE Communications Magazine, vol. 50, no. 2, pp. s58-s66, Feb. 2012.

[2] A. S. Karar and J. C. Cartledge, "Generation and detection of a 112-Gb/s dual polarization signal using a directly modulated laser and halfcycle 16-QAM Nyquist-subcarrier-modulation,” in European Conference on Optical Communication (ECOC), 2012, p. Th.3.A.4.

[3] R. Rodes, J. Estaran, B. Li, M. Mueller, J. B. Jensen, T. Gründl, M. Ortsiefer, C. Neumeyr, J. Rosskopf, K. J. Larsen, M.-C. Amann, and I. T. Monroy, “100 Gb/s single VCSEL data transmission link," in Optical Fiber Communication, National Fiber Optic Engineers Conference (OFC/NFOEC), 2012, p. PDP5D.10.

[4] T. Tanaka and M. Nishihara, "Experimental investigation of 100-Gbps transmission over 80-km single mode fiber using discrete multi-tone modulation," in SPIE 8646N, 2013, vol. 8646.

[5] D. D. Falconer, "Carrierless AM/PM," 1975.

[6] A. F. Shalash and K. K. Parhi, "Comparison of discrete multitone and carrierless AM/PM techniques for line equalization," in IEEE International Symposium on Circuits and Systems. (ISCAS), 1996, vol. 2, pp. 560-563.

[7] N. G. Gonzalez, D. Zibar, X. Yu, and I. T. Monroy, "Optical phase-modulated radio-over-fiber links with k-means algorithm for digital demodulation of 8PSK subcarrier multiplexed signals,” in Optical Fiber Communication, National Fiber Optic Engineers Conference (OFC/NFOEC), 2010, vol. 1, p. OML3.

[8] I. Dedic, "High-speed CMOS DSP and data converters," in Optical Fiber Communication, National Fiber Optic Engineers Conference (OFC/NFOEC), 2011, p. OTuN1. 